\title{
La gestión de las políticas de fomento del empleo juvenil en el ámbito municipal: resultados de una investigación
}

\author{
M.J. Aguilera Arilla* ${ }^{*}$ M.V. Azcárate luxán*, M.P. Borderías Uribeondo*, \\ M.P. GonzÁlez YanCI* Y J.M. Santos PReCIADO*
}

\section{RESUMEN SUMMARY}

La evaluación de las políticas de inserción laboral de los jóvenes en el ámbito territorial municipal, centradas en

la eficacia de la formación ocupacional no reglada, nos ha senvido para reflexionar sobre la capacidad de este instrumento operativo en la adecuación de la oferta y la demanda laborales. Este articulo supone un amplio resumen de los resultados obtenidos, de acuerdo a esta línea de investigación, en un trabajo recientemente realizado en el municipio de Getafe.
The evaluation of the policies for introduction of young people to their first employment in towns, centred in the efficiency of occupational unregulated training, has served us to reflect on the capacity of this operational tool to match up supply and demand for young workers. This article provides a comprehensive summary of the results obtained from this line of investigation in a study recently undertaken in the municipality of Getafe.

\section{INTRODUCCIÓN}

$\mathrm{El}$ análisis de los desequilibrios del mercado laboral juvenil, en el espacio metropolitano madrileño, realizado, en un trabajo previo, por el mismo equipo investigador (Aguilera Arilla, M.J. y otros, 1996), nos hizo reflexionar sobre la trascendencia del territorio municipal en la aplicación de cualquier política de empleo. La canalización de recursos y el control de

* U.N.E.D., Madrid. 
iniciativas, en materia de formación profesional y fomento del empleo, suponíamos que mejoraría en este ámbito territorial, debido al conocimiento del entorno local y a la proximidad de los ciudadanos, principales agentes activos beneficiarios de toda política territorial.

Por este motivo, el objetivo fundamental del proyecto de investigación, que recientemente hemos finalizado (Aguilera Arilla, M.J. y otros, 1999), cuyos resultados presentamos, ha sido el de analizar la problemática de la inserción laboral de los jóvenes y las políticas específicas que tal propósito conlleva en el ámbito municipal, para lo cual hemos seleccionado el municipio de Getafe como lugar idóneo donde realizar un estudio de esta naturaleza.

La elección del municipio de Getafe, como banco de pruebas de observación de la realidad, no ha sido en absoluto baladí; fundamentalmente, por estar ubicado en una de las zonas más problemáticas de la región metropolitana madrileña. Los resultados obtenidos en nuestra investigación anterior identificaban el sur Area Metropolitana de Madrid, como una de los espacios con más dificultades para la inserción laboral juvenil. Los jóvenes residentes en este espacio geográfico presentan las tasas de desempleo más elevadas, en consonancia con su bajo nivel formativo. Además, la importancia de este sector metropolitano, en cuanto a la problemática laboral se refiere, se deriva del elevado porcentaje de jóvenes respecto al total del área metropolitana (aproximadamente el 50\% del total).

El estudio minucioso del desarrollo de las políticas de formación ocupacional en Getafe se ha centrado en el seguimiento profesional de los jóvenes que habían participado en los cursos y programas organizados desde el propio ayuntamiento de la localidad. Sin embargo, una tarea de la naturaleza de la propuesta evidenciaba la necesidad de alcanzar otros objetivos preliminares: un estudio previo sobre el colectivo de los jóvenes de la localidad, que pusiera de manifiesto la problemática de su inserción laboral, así como el análisis detallado del marco legislativo, desagregado territorialmente, desde el que se ofrecían soluciones a los problemas planteados.

\section{LOS OBJETIVOS FUNDAMENTALES DE LA INVESTIGACIÓN}

Los objetivos concretos de la investigación realizada los hemos agrupado en tres áreas específicas, con carácter propio:

1) En primer lugar, se han analizado las características generales del colectivo juvenil en el municipio de Getafe (demográficas, sociales, formativas, etc.), completando esta descripción global con la elaboración de una 
tipología de jóvenes, que pusiera de manifiesto la existencia de problemáticas laborales diferenciadas.

El mercado de trabajo juvenil puede ser considerado como un segmento del mercado laboral general, con unas características propias. Pero incluso desde esta perspectiva, no constituiría un todo unitario. La fragmentación social del mundo laboral afecta, así mismo, a los jóvenes, permitiendo la identificación de sectores de población de características muy contrastadas. Así, junto a jóvenes en paro o con trabajos en precario, sobreviviendo de la economía sumergida, se hallan otros con empleos estables y bien remunerados o jóvenes empresarios con capacidad e iniciativa para crear empresas de éxito social. Diversas variables (sexo, edad, nivel de instrucción, posición frente a la actividad laboral, etc.) resultan fundamentales en la intención de delimitar submercados laborales-tipo. Estos tipos ideales, construidos según el criterio de investigación sociológica de A.Weber, tienen sentido como arquetipos de la realidad social que intentan representar. Su identificación ha facilitado el estudio posterior de las diferentes políticas juveniles de integración en el mundo del trabajo, al permitir un análisis diferenciado para cada grupo social específico.

2) El segundo objetivo de la investigación ha estado dirigido al estudio general y pormenorizado de las políticas de empleo juvenil desarrolladas en el municipio, tratando de establecer un marco institucional (a diversas escalas territoriales, internacional, estatal y autonómico), que permitiera obtener una panorámica de las posibilidades de actuación en este campo sectorial específico. Las medidas de fomento del empleo han sido analizadas desde las dos principales vías de actuación: creación directa o indirecta de empleo juvenil y formación profesional ocupacional.

3) El tercer objetivo de la investigación, que considerábamos fundamental, estaba dirigido a la evaluación detallada de los resultados alcanzados con las políticas de formación ocupacional, desarrolladas por el conjunto de programas y cursos gestionados en el ámbito municipal.

Gran parte de los estudios realizados, en esta área concreta de la investigación, han consistido en análisis parciales sobre el éxito o fracaso de la formación impartida, pero más con criterios cuantitativos globales (quiénes han encontrado trabajo al final de los cursos) que cualitativos (si el trabajo encontrado tiene relación con la formación recibida). La realización de un seguimiento detallado de las relaciones múltiples existentes entre las características personales de los receptores de la formación (demográficas, sociales y de nivel de instrucción), particularidades de los cursos impartidos (diferenciados por tipos de programas) y grado de reciprocidad existente entre la formación recibida y el puesto de trabajo conseguido ha 
permitido plantearse, entre otros, un importante conjunto de cuestiones relativas al éxito o fracaso de los programas de formación impartidos, así como al nivel de integración de la mano de obra juvenil en el mercado de trabajo local y a las necesidades de formación reales que el mercado de trabajo demanda.

\section{RESULTADOS OBTENIDOS}

Los principales resultados del trabajo realizado los vamos a exponer, de acuerdo a los tres objetivos fundamentales de la investigación:

\subsection{Características del colectivo juvenil de Getafe. Tipologías} de jóvenes, según la problemática de la inserción laboral

El estudio detallado de las características formativas y socioprofesionales de los jóvenes de Getafe, como paso previo al análisis de las políticas de empleo implementadas en el municipio, nos ha servido para, con carácter general, definir las necesidades laborales juveniles, existentes en uno de los espacios geográficos metropolitanos más representativos de la dificultad de integración en el mundo del trabajo. Algunas de las cualidades analizadas son fruto de la coyuntura (recuperación de la economía española), mientras que otras poseen un carácter estructural. El análisis diferencial, en el espacio (respecto a la Comunidad de Madrid) y en el tiempo (durante el periodo de 1991 a 1996), reproduce la especificidad del mercado laboral juvenil, tal como había sido definido en el apartado anterior.

Las conclusiones de mayor interés a este respecto han sido las siguientes:

- Durante los últimos años, se ha producido un notable incremento de las tasas de escolaridad juvenil en todo el territorio madrileño. Esta superior escolarización, que es el fruto de las respuestas ofrecidas desde el sistema institucional a la dificultad de la inserción laboral, se manifiesta, de manera sustancial, en el escalón de edad comprendido entre los 16 y 19 años y, en menor medida, aunque importante, en el de 20 a 24 años (en Getafe, el crecimiento, mayor que en la CAM, ha sido de $11.28 \%$ y $8.13 \%$ respectivamente).

- El consecuente retraso en la integración al mercado laboral no deja de presentar aspectos positivos, como la mejora del nivel de instrucción y de estudios (en los años transcurridos entre 1991 y 1996, Madrid ha dis- 
minuido en 9 puntos el porcentaje de jóvenes de edad comprendida entre 20 y 29 años, que únicamente tienen estudios primarios o no tienen ningún estudio, y Getafe en 4.5). Asimismo, el porcentaje de titulados universitarios de segundo y tercer ciclo, en el escalón de 25 a 29 años, ha crecido, en idéntico período de tiempo, un $10.73 \%$ y un $3.08 \%$ respectivamente.

- La incorporación a la actividad económica se ha visto afectada por factores de carácter contrapuesto. Mientras que el aumento de la escolarización de los más jóvenes explica el acentuado descenso de las tasas de actividad de este grupo de edad, así como del porcentaje de trabajadores parados en busca del primer empleo, el crecimiento de la mano de obra femenina, como consecuencia de la recuperación económica, ha disparado la tasa de actividad de las mujeres casadas mayores de 20 años. El hecho ha resultado concluyente en Getafe, donde la tasa de actividad femenina de las mujeres no solteras ha subido el $10,36 \%$, en el escalón de 20 a 24 años, y el 12,18\% en el correspondiente al de 25 a 29 años.

- La tendencia a la terciarización de la economía española se refleja en la evolución de la estructura de la población activa por ramas productivas. Esta disposición es más pronunciada en las mujeres que en los hombres, habiéndose incrementado la diferencia, por sexo, al $27 \%$ en el territorio de Getafe (únicamente del $20 \%$ en la CAM).

- La especialización ocupacional se manifiesta en los perfiles profesionales de los jóvenes trabajadores, que adquieren una especificidad, relacionada con el sexo y la edad, como consecuencia de la selección que determina el mercado laboral. Esta selección está marcada, además, por el nivel socioeconómico, lo que resulta definitivo en el nivel formativo adquirido y en el momento de abandono del sistema educativo. Así, el colectivo de jóvenes de menor edad relativa se halla especializado en profesiones de baja cualificación y reducido grado de instrucción (trabajadores manuales y no cualificados, destacando la participación del varón en la construcción y de la mujer en los servicios). Por su parte, el grupo de mayor edad relativa destaca por la ocupación en aquellas profesiones de mayor cualificación (titulados superiores y medios, con mayor concentración masculina en las de carácter técnico y científico y de la mujer en las ramas de Sanidad, Educación y Cultura), de responsabilidad, iniciativa y mando (propietarios, directores, jefes de departamento, jefes de venta, contramaestres, etc., mayoritariamente ocupadas por hombres) o empleados de nivel medio-bajo (auxiliares administrativos, auxiliares de enfermeria, empleadas del hogar, etc., mayoritariamente ocupadas por mujeres).

- El estudio territorial, diferenciado por barrios y secciones censales, muestra la existencia, dentro del municipio de Getafe, de espacios so- 
ciales, de características contrastadas, pertenecientes a mercados de trabajo diferentes. Así, existirian dos áreas, coincidentes casi plenamente con los barrios del Sector 3 y Getafe Norte, de expansión reciente, altamente correlacionadas con las variables propias de un mercado laboral primario (alta tasa de escolaridad, tardio abandono del sistema escolar, elevado porcentaje de titulados universitarios, alta tasa de actividad femenina del escalón de 25 a 29 años, menor tasa de actividad masculina de los jóvenes menores de 24 años y unos perfiles de ocupación en las profesiones de mayor rango). En el extremo opuesto, se hallarían tres áreas de menor categoría social (barrios de la Alhóndiga, San Isidro y Las Margaritas), relacionadas con las características de un mercado laboral secundario (baja tasa de escolaridad, temprano abandono del sistema educativo reglado, baja tasa porcentual de titulados universitarios y de actividad femenina de las mujeres de mayor edad, mayor tasa de actividad de los jóvenes de edad reducida y perfiles profesionales de menor rango, destacando los trabajadores no cualificados y de la construcción). Además, existirian dos áreas de nivel medio-bajo (barrios de Juan de la Cierva y El Bercial) y un área heterogénea, con fuertes contrastes geográficos internos, que se corresponde con el barrio Centro del municipio.

Finalmente, la aplicación de una metodología particular, sobre la hipótesis de la existencia de arquetipos de jóvenes, desde la perspectiva de los procesos de transición del mundo de la escuela al del trabajo, en el municipio de Getafe, nos ha permitido identificar itinerarios específicos, relacionados con la edad, el sexo, el entorno social, la formación académica recibida y la experiencia profesional. Al menos cuatro colectivos de jóvenes han podido ser deducidos del análisis realizado: jóvenes fracasados en el sistema escolar, de difícil inserción laboral, jóvenes del entorno obrero, con un relativo nivel de formación, que se incorporan tempranamente al sistema educativo, jóvenes de nivel de estudios medios o superiores (universitarios) y mujeres:

- El primer perfil socioprofesional corresponde a los jóvenes más problemáticos. Constituye, sin duda, el subgrupo de población juvenil de más difícil inserción en el mercado laboral. Su temprano abandono del sistema educativo reglado conlleva un escaso nivel formativo, lo que unido a su reducida o nula experiencia laboral incrementa las naturales dificultades de encontrar trabajo. La gran rotación del empleo juvenil es superior, incluso, en este segmento de población, abocado a desempeñar tareas subalternas, de escasas perspectivas profesionales. Su frustración es, en consecuencia, muy grande y a ellos van dirigidas gran parte de las politicas de empleo juvenil. 
- El denominado modelo obrero, como arquetipo de la independencia juvenil en el medio proletario, quedaba definido, en el varón, por el paso casi instantáneo de la infancia a la edad adulta y la concordancia en el tiempo del desarrollo activo de la vida profesional, el matrimonio y el abandono del hogar paterno. Hoy en día, el modelo ha desaparecido en sentido estricto, pero conserva algunos rasgos específicos, especialmente los derivados del abandono de la escuela y la incorporación al mundo del trabajo. En muchas familias obreras, la integración a corta edad en la "fábrica" o en el "tajo" es valorada positivamente, al adivinar en la continuidad de los estudios una doble pérdida: la de adquirir una experiencia profesional y la de ingresar los emolumentos económicos generados por el trabajo. Este colectivo de jóvenes revela, en gran parte, su procedencia de esta clase social. Constituye un subgrupo homogéneo, que a pesar de su bajo nivel de instrucción, valora positivamente su pertenencia al mundo del trabajo, sobretodo, por el orgullo del oficio desempeñado.

- El grupo de estudiantes universitarios ocupa una posición privilegiada en el mercado laboral, como resultado de su superior nivel de instrucción. Su retraso en la incorporación al mundo del trabajo no deja de presentar aspectos formativos positivos, aunque suponga un alejamiento psicológico de la realidad laboral y una tardía emancipación del núcleo familiar. Sus mejores expectativas de encontrar empleo se explican por varias razones. Por un lado, la reestructuración industrial del sistema productivo (reconversión, cambio tecnológico, etc.) está demandando un personal activo de mayor nivel de cualificación, mientras que, por otro, la ocupación en subempleo, de puestos laborales de nivel inferior, les permite acceder a trabajos tradicionalmente reservados a personas de formación inferior.

- Finalmente, el colectivo femenino, a pesar de la mejora de su nivel educativo, continúa estando discriminado en el mercado laboral. Comparativamente, las profesiones y oficios femeninos presentan menor grado de cualificación profesional, un salario más bajo y menores oportunidades de ascenso que los masculinos. Sin embargo, actualmente, estamos asistiendo, a un cambio profundo de las condiciones de trabajo que afectan a la mujer, pese al desfase que todavía continúa existiendo. Hasta hace relativamente poco tiempo, el trabajo de la mujer se centraba, mayoritariamente, en la ayuda familiar, el comercio, la hostelería, el servicio doméstico y determinadas ramas industriales, como la alimentación y el textil. Hoy en día, su nivel profesional ha sufrido un notable incremento, lo que se ha visto reflejado en el gran número de tituladas medias y superiores, que vienen desarrollando su profesión, fundamentalmente, en el sector servicios, en las ramas de la educación, la sanidad y la administración pública en general. Pese a estas favorables perspectivas, cualquier 
política de empleo juvenil que se precie debe considerar la mejora de la posición de la mujer, facilitándola la consecución de puestos de trabajo tradicionalmente reservados a los varones, superando los obstáculos de discriminación y segregación existentes.

\subsection{Las políticas de fomento del empleo juvenil. La aplicación de estas políticas en las diversas escalas territoriales}

Las políticas generales de fomento del empleo juvenil

La mejora de las condiciones económicas generales de un país, con ser fundamentales, no siempre garantiza, por la sola actuación de las leyes del mercado, la creación de puestos de trabajo para los colectivos sociales más desfavorecidos. Un conjunto de medidas favorece la acomodación de la mano de obra juvenil a las necesidades objetivas del mercado de trabajo: desarrollo del sistema educativo formal; mejora de la formación profesional ocupacional; e incremento de la oferta de empleos destinados a los jóvenes.

El sistema educativo reglado constituye la primera etapa de la preparación curricular de los jóvenes, en la perspectiva de su futura integración a la vida profesional activa. En nuestro país, el cierre del sistema educativo sobre si mismo, ajeno a las necesidades demandadas por el sistema productivo, ha propiciado el olvido de una formación para el trabajo. La dificultad de acomodar formación educativa y desarrollo profesional, obliga a ser cautos a la hora de ofrecer soluciones fáciles en un campo tan complejo. La conclusión más sensata apunta a la necesidad de definir una política educativa flexible, que tenga presente la existencia de un mundo en constante transformación que, cada vez con más frecuencia, va a exigir el cambio de oficio a lo largo de la vida profesional.

La transición de la escuela al mundo del trabajo supone un reto, cuyo resultado puede ofrecer interesantes soluciones al problema de la inserción laboral de los jóvenes. El modelo de “formación en alternancia», que enlaza con el modelo dual alemán, al pretender combinar la formación en los centros educativos con la realización de prácticas en las empresas, supone una línea de integración educativa y laboral que debería profundizarse, sin importar los esfuerzos requeridos.

Pero, incluso, considerando adecuado el nivel de instrucción recibido en las instituciones educativas, la constante reestructuración del sistema productivo exige requisitos de formación continua de los trabajadores. Las 
innovaciones tecnológicas del momento presente demandan, además, nuevos perfiles de mano de obra, lo que obliga a la Administración a intervenir en la oferta de una formación profesional suplementaria.

Las soluciones posibles en el campo de la formación de la mano de obra juvenil son múltiples. En este sentido, la formación ocupacional no reglada se revela como una actividad con una alta capacidad potencial para incidir positivamente sobre los problemas laborales actuales. Este tipo de formación profesional es una vía necesaria para la recualificación profesional de los trabajadores que abandonaron el sistema escolar y, estando incorporados a la actividad laboral, necesitan un reciclaje, acorde con las necesidades del empleo que ocupan. Esta intervención se hace más necesaria si cabe, respecto a los jóvenes que abandonan el sistema escolar a pronta edad, porque su nivel formativo resulta más precario, lo que dificulta su integración al trabajo.

Finalmente, otro tipo de medidas está destinado a mejorar la oferta de trabajo juvenil, bien por la vía de redistribuir la misma en beneficio de los más jóvenes, bien por la creación de una nueva oferta suplementaria a la actualmente existente, bien por la creación con dinero público de puestos de trabajo para los jóvenes

La dificultad de la incorporación laboral de los jóvenes reside en la competencia que, frente a ellos, ofrecen colectivos laborales de mayor nivel de cualificación y que, en las circunstancias del momento presente (flexibilidad laboral, salarios próximos al mínimo legal establecido, etc.), concurren con mayores posibilidades de éxito. La justificación económica para la contratación de una persona joven tendría que venir, bien por el camino de una reducción de su salario, bien por la subvención pública de este tipo de empleo. Esta última vía ha sido la más utilizada, desde las administraciones autonómica y estatal para la solución del problema.

El segundo tipo de proposiciones se ocupa del desarrollo del sistema productivo, con la esperanza de que un mayor dinamismo empresarial favorezca la creación del empleo en general, y del dirigido a los jóvenes, en particular. En esta línea de trabajo, una actividad interesante ha sido la aplicación de políticas encaminadas a estimular el autoempleo e impulsar las iniciativas juveniles en el campo empresarial.

Finalmente, la creación de empleo desde el sector público constituye el tercer medio para incrementar la demanda de jóvenes trabajadores. Este tipo de soluciones puede resultar interesante, aunque limitadas, en una sociedad de libre mercado como la nuestra. En estas circunstancias, los Ayuntamientos suelen ser una de las principales empresas en sus respectivos municipios, lo que permite plantearse la posibilidad de fomentar 
las contrataciones de los jóvenes que presentan más problemas para su inserción laboral.

La aplicación de las políticas de fomento del empleo juvenil, a diversas escalas territoriales

La aplicación de las políticas de fomento del empleo juvenil, anteriormente descritas, se ve condicionada por el complejo entramado de ámbitos de poder territorial. En España, cuatro niveles jerárquicos inciden en la toma de decisiones espaciales: el europeo, el estatal, el autonómico y el municipal, dificultando la coordinación y ejecución de este tipo de políticas.

En el escalón territorial transnacional, la adhesión española a la Unión Europea ha supuesto una importante aportación económica a la hora de estimular la resolución del problema del desempleo juvenil, promoviendo, fundamentalmente, la formación profesional de los jóvenes, con la intención de facilitar el acceso al trabajo. Desde esta perspectiva, el Fondo Social Europeo ha orientado las políticas de empleo hacia los colectivos sociales más desfavorecidos, como el que nos ocupa.

A través de diferentes programas de iniciativa comunitaria: EMPLEO, ADAPT, SOCRATES, LEONARDO, EMPLOYMENT, etc., se han articulado las políticas de ayuda al desarrollo del empleo y formación profesional ocupacional juvenil, destinadas, fundamentalmente, a los jóvenes que carecen de cualificación suficiente y, también, las que intentan lograr la igualdad de oportunidades para el acceso de la mujer al mercado laboral.

Esta importante inyección económica, que superó en 1997, en nuestro país, la cifra de 250.000 millones de pesetas, y en la CAM la de 23.000 millones, ha determinado que muchas de las iniciativas de formación y empleo puedan llevarse a efecto gracias a la financiación procedente de la UE, teniendo presente que España se encuentra a la cola de Europa, en cuanto a inversión en formación de los trabajadores.

En el ámbito estatal, la implementación de las políticas de empleo juvenil se realiza a través del INEM, organismo encargado de la gestión y coordinación de las mismas en el conjunto del territorio nacional. Colaboran, además, otros organismos, como el Ministerio de Educación y Ciencia, el Instituto de la Mujer, etc.

Entre las medidas previstas para la creación de empleo, destacan aquellas dirigidas al sector privado de la economía, de carácter legislativo (contratos para la formación, en prácticas, a tiempo parcial, etc.) o de incentivación económica (subsidios, ventajas fiscales, bonificaciones a la 
Seguridad Social, etc.). Sin embargo, en el sector público, es donde la intervención de la Administración, mediante la creación directa de empleo o el fomento indirecto del mismo (apoyo a las cooperativas y el autoempleo, iniciativas locales de empleo, etc.), puede desarrollar e incrementar los puestos de trabajo dirigidos a los jóvenes de más difícil inserción laboral.

Sin embargo, la intervención del INEM ha resultado determinante en el terreno de la formación. La puesta en marcha del Plan Nacional de Formación e Inserción Profesional (FIP), a partir de 1985, ha potenciado las acciones formativas dirigidas a los jóvenes parados, cuya cualificación profesional resulta en la práctica insuficiente $O$ inadecuada. El plan FIP, desde un punto de vista cuantitativo, ha supuesto un fuerte crecimiento de las acciones formativas, mientras, cualitativamente, va cumpliendo sus objetivos prioritarios de dirigir la formación hacia los colectivos sociales más desfavorecidos.

Otra de las iniciativas formativas del mayor interés la constituyen los programas de Escuelas Taller y Casas de Oficios. Creados por el Ministerio de Trabajo, a través del INEM, combinan el aprendizaje de una profesión con el trabajo productivo y están dirigidos a jóvenes desempleados que, o no han conseguido finalizar sus estudios de EGB o, habiéndolos terminado, no continúan estudiando.

Además, los programas de Garantía Social son desarrollados, fundamentalmente, por el Ministerio de Educación y Ciencia, proporcionando a los jóvenes, de edad comprendida entre 16 y 21 años que hayan fracasado en el sistema educativo reglado (sin titulación académica superior al graduado escolar) y sean demandantes, prioritariamente, del primer empleo, una formación básica y profesional, que les permita, mediante el aprendizaje de un oficio, incorporarse a la vida activa.

Finalmente, el Instituto de la Mujer, dependiente del Ministerio de Asuntos Sociales, realiza diversas actuaciones de fomento del empleo y de formación ocupacional de la mujer, con la finalidad de mejorar su presencia en el mundo del trabajo, favoreciendo el cambio de hábitos que dificultan su inserción laboral en condiciones de igualdad con los varones.

Todos estos programas formativos, organizados desde organismos de carácter nacional, son, sin embargo, gestionados, en parte, en la impartición de sus enseñanzas, por otros organismos, públicos (comunidades autónomas, ayuntamientos, sindicatos, universidades, institutos sociales, etc.) o privados, lo que afecta a un conglomerado de instituciones, dificultando la evaluación de los resultados alcanzados.

En los últimos años, de forma progresiva, las comunidades autónomas han ido recibiendo competencias en materia de formación y empleo. 
Centrándonos en la CAM, ambas iniciativas se canalizan a través de determinadas consejerías. Así, la Consejería de Economía y Empleo resulta clave en el Plan de Empleo, ya que, a partir de la Dirección General de Empleo, concede ayudas para nuevas iniciativas empresariales, para proyectos generadores de empleo, para formación y para fomento del desarrollo local.

En el campo de la formación profesional ocupacional, dicha Consejería cuenta con una interesante oferta de cursos formativos. Destaca, amén de otros organismos anejos (Consejerías de Sanidad y Servicios Sociales, de Educación y Cultura, y de Medio Ambiente y Desarrollo Regional), la actuación del Instituto Madrileño para la Formación (IMAF), por la ingente labor desarrollada en esta área específica de la política de fomento del empleo, en general, y juvenil, en particular. En la programación de sus actividades formativas participan, tanto las organizaciones sindicales y empresariales, como la Comisión Europea, que cofinancia los cursos a través del FSE, el INEM y los ayuntamientos de la Comunidad de Madrid. EI IMAF contempla la formación específica de determinados colectivos (mujeres, jóvenes en busca del primer empleo, etc.), por lo que su actuación resulta esencial en la solución del problema de la inserción laboral juvenil.

La política local constituye el marco de actuación más inmediato y próximo al ciudadano, lo que permite movilizar los factores de desarrollo municipal, con vistas a dinamizar y recualificar su propio mercado de trabajo.

En el municipio de Getafe, las iniciativas a favor del empleo han seguido idénticas vías a las descritas en los otros ámbitos territoriales: fomento del empleo y de la formación. En la primera área de actuación, las actividades desarrolladas han sido múltiples: promoción de suelo industrial, asesoría a nuevos emprendedores, activación de las medianas y pequeñas empresas, formación empresarial, fomento de la adaptación del tejido empresarial a los cambios tecnológicos, observatorio socioeconómico del municipio (seguimiento, análisis y diagnóstico de la evolución de la economía), etc. Todas estas iniciativas pretenden el desarrollo de recursos locales y la adaptación de las empresas del entorno local a los retos de la sociedad de la información, fomentando cualquier actividad personal de espíritu emprendedor, en la línea de creación de nuevas empresas; amén, de servir de organismo intermediario entre la oferta y la demanda laborales.

En el campo de la formación, no vamos a repetir la importancia de la formación profesional ocupacional (FPO), como vía de recualificación de la mano de obra y potenciación del empleo. La variedad de los programas 
(Garantía Social, Escuelas Taller, Casas de Oficios, Plan FIP, IMAF, Específicos de la Mujer, etc.) y de los cursos impartidos por el ayuntamiento de Getafe, en colaboración con otros organismos nacionales (INEM, IMAF, MEC, Instituto de la Mujer, etc.) e internacionales, revela el amplio abanico de opciones formativas, con vistas a facilitar la inserción laboral de colectivos sociales con problemática muy dispar.

La gestión integrada del empleo y la formación se ve favorecida en el entorno local, al permitir observar, directamente, la aplicación y seguimiento de los diversos programas de empleo y formación. Esta labor requiere que las acciones formativas vayan acompañadas de otras complementarias, de orientación, conexión con el mundo laboral de las empresas y seguimiento de la validez de los cursos impartidos.

La importancia que ha adquirido el área de la formación y el empleo en un municipio de la dinámica productiva del de Getafe exige la organización administrativa de la corporación local, en la línea de una mayor eficacia de la gestión. La creación de organismos independientes, como el Organismo Autónomo Formación-Empleo, o la empresa GISA sirven para centralizar la gestión, tratando de integrar las competencias de formación y empleo a escala local, con la finalidad de potenciar la coordinación con otras instituciones (autonómicas, estatales e internacionales).

Las medidas de fomento del empleo son diversas y su evaluación global permite deducir una serie de conclusiones de carácter general, que matizaremos, en el aspecto formativo, al referirnos al análisis de las políticas de formación ocupacional gestionadas en el ámbito municipal.

Como primera conclusión general, si bien consideramos que la formación profesional no es ninguna panacea, por la existencia de un paro estructural de difícil solución, podemos inferir que la incorporación de la misma a las políticas de fomento del empleo ha resultado ser un instrumento de ayuda a la movilidad ocupacional y a la capacidad de hacer frente al cambio tecnológico del momento actual. Así mismo, ha resultado ser un elemento operativo en la búsqueda de igualdad de oportunidades para los colectivos sociales más desfavorecidos.

La intervención de las distintas administraciones, a favor de la inserción laboral de los jóvenes, requiere la mejora de la coordinación entre los distintos escalones territoriales, que propicie una mayor efectividad de las distintas actuaciones en esta materia. Esta articulación entre los niveles nacional, regional y local debe vincularse a un movimiento de descentralización administrativa, que potencie la iniciativa de los dos últimos escalones mencionados. En esta línea de actuación, el conocimiento del mercado de trabajo, a partir de las necesidades de las empresas y de las nuevas 
tecnologías a implantar, debe definir los perfiles profesionales a los que se debe ajustar la oferta formativa. En este sentido, el conocimiento, por parte de los ayuntamientos, de los mercados locales de empleo, a partir de sus observatorios ocupacionales, resulta fundamental a la hora de diseñar los programas de formación.

Esta labor se puede complementar con la participación conjunta de todos los agentes que intervienen en el proceso de inserción laboral (empleadores, educadores, sindicatos, individuos, etc.). En este sentido, pueden resultar de interés actividades como la organización de jornadas técnicas para el análisis de las tendencias del mercado de trabajo, que pueden arrojar luz sobre el panorama laboral de los desajustes existentes entre las características de la mano de obra y las necesidades del sistema productivo.

Por otro lado, la flexibilidad laboral, como instrumento de promoción del empleo, fomentada, incluso, por medio de ayudas económicas de diferente condición (subvenciones, ventajas fiscales, bonificaciones, etc.), debe ser cuestionada, al menos, por el desequilibrio existente entre las ventajas que ofrece al empleador frente al trabajador. Son, los contratos temporales, precisamente, los que propician la inestabilidad y precariedad de las condiciones de trabajo de los jóvenes menos cualificados profesionalmente, lo que dificulta su integración laboral. Por este motivo, se deben primar aquellas políticas en las que las ayudas al empresario vayan acompañadas de una estabilidad en el empleo, que garantice la formación permanente y el aprendizaje de una profesión.

Resulta, además, de gran transcendencia la existencia de contactos y convenios entre los programas de formación y las empresas, como futuro vehículo de inserción en el mundo del trabajo. Esta relación se debe profundizar, desde el propio sistema educativo reglado, mediante la «formación en alternancia», que procura la complementariedad de la acción formativa, mediante la realización de prácticas en las empresas. En mayor medida, la conexión debe existir en la formación ocupacional, más próxima a la incorporación al mundo del trabajo. Por este motivo, no se debe olvidar, que la acción formativa no termina con la realización de los cursos, sino que se debe continuar prestando servicios a los alumnos, a través de acciones de acompañamiento al empleo: orientación sociolaboral; prácticas profesionales en empresas; atención a empresas en demanda de alumnos formados; y seguimiento de la inserción. Y aún más, los programas de formación deberían prever la posibilidad de que parte de la acción formativa se verificara en el seno de las propias empresas. De esta manera, el acercamiento entre la oferta y la demanda de formación tendria unos frutos más duraderos y definitivos. 


\subsection{La valoración de las políticas de formación profesional ocupacional, desarrolladas por el Ayuntamiento de Getafe}

Dentro de las posibilidades de exploración del mercado de trabajo juvenil, elegimos, como medio de valoración, la aproximación al colectivo objeto de estudio, mediante encuesta. Las limitaciones de los resultados obtenidos, al tener que aplicarse a una muestra, se ven, sin embargo, recompensadas por el amplio conocimiento que un estudio de esta naturaleza proporciona.

La encuesta se realizó a un grupo de jóvenes, seleccionados por su participación en los cursos de formación ocupacional, organizados por el Ayuntamiento de Getafe. El colectivo resultante estaba compuesto por un total de 363 personas, todas ellas entre 16 y 29 años, equilibrado en cuanto a su distribución por sexo, aunque con un ligero predominio de varones $(52,3 \%)$. Sus características sociodemográficas muestran una mayoría de solteros, que vivían con sus padres, aunque manifestaban no depender económicamente de nadie, que sólo en muy reducida proporción tenían a su cargo hijos o padres y que no mostraban grandes deseos de independencia. A partir de los estudios y ocupación de los padres, las familias de estos jóvenes pueden considerarse predominantemente de clase media baja. Los padres tenian, en general, tan sólo estudios elementales, dándose entre las madres incluso casos de analfabetismo. Desde el punto de vista laboral, destacan las altas tasas de inactividad en las madres y el predominio de obreros, especializados o no, entre los padres. Todos los rasgos citados, unidos a la existencia entre los padres de un número considerable de inactivos (jubilados, parados y amas de casa) parecen mostrar una situación poco favorecida en relación con el conjunto de la sociedad.

El nivel de formación adquirido por los jóvenes en enseñanzas regladas es muy variado. Hay que tener en cuenta dos hechos relevantes: la edad, que condiciona los estudios que han podido concluir, y la especificidad de alguno de los cursos impartidos por el Ayuntamiento de Getafe, como el dirigido a mujeres licenciadas. Estos hechos, evidentemente, producen un sesgo en los resultados. Pese a ello, podemos extraer como conclusión general, que el nivel de formación adquirido era bajo (cerca del $40 \%$ tenían estudios primarios y graduado escolar). Existía, además, un notable contraste entre hombres y mujeres, siendo más numerosos los varones con estudios inferiores $y$, en cambio, las mujeres destacaban en los niveles de estudios más altos. Se apreciaba, además, una notable mejora de la formación de los jóvenes con respecto a sus padres, especialmente en el caso de las mujeres. 
El análisis de las edades de abandono y de finalización de estudios mostraba un cierto grado de fracaso escolar, manifestado, por un lado, en la salida precoz del sistema educativo y, por otro, en la tardía edad de finalización de estudios con respecto a la edad oficial esperada. Los motivos por los que abandonaron los estudios clarifican esta aseveración, ya que la mayoría de los que pertenecían a los niveles inferiores declararon que no les gustaba estudiar. Se detecta que no era la motivación económica la que más impulsaba a abandonar los estudios y que entre las mujeres existía una mayor proporción que dejaban de hacerlo por una pretendida búsqueda de independencia.

La correspondencia entre lo que han conseguido y lo que quisieran ser se da en pocos casos. Los deseos de la mayoría estribaban en tener una profesión tradicional o carrera universitaria, siendo muy pocos los jóvenes que anhelaban desarrollar profesiones creativas, novedosas o que muestren espíritu de empresa.

La mayoría complementó su formación con estudios no reglados. Predominaban los de mecanografía, informática e idiomas, lo que denota que es generalizada la necesidad que sienten de completar la enseñanza "oficial y reglada".

A partir de la formación académica expuesta, los jóvenes demandaban participar en diversos cursos de formación ocupacional, cursos que realizaron entre 1995 y 1997, que eran sumamente variados, agrupados en siete programas, en los que eran mayoría los relacionados con oficios tradicionales. Además, en muchos casos, realizaron cursos en otros organismos oficiales o subvencionados, con idéntico contenido temático, lo que demuestra una gran predisposición para mejorar su formación, de cara a su integración en el mundo laboral.

La inscripción en el INEM nos informa sobre las capacidades profesionales potenciales, que los propios alumnos creian poseer. Un elevado porcentaje $(59 \%)$, manifestó una profesión o demanda de empleo concreto, representando a los que, o bien habian trabajado ya en esas profesiones, o se sentían capacitados, o con deseos de realizar esos trabajos. La mayoría de los que expresaban una profesión correspondia a trabajadores manuales, tanto de la industria como de la construcción o servicios, aunque por separado eran los administrativos los más numerosos. A la hora de inscribirse en el INEM para demandar empleo, se manifiestan diferencias entre hombres y mujeres, según los estudios previos que poseyeran, destacando los siguientes hechos: los hombres se inscribían más como trabajadores manuales de industria y construcción, mientras que las mujeres preferian hacerlo como administrativas. En ambos casos, existian 
múltiples situaciones donde la demanda de trabajo era independiente de su nivel de formación (se da el caso de hombres titulados que se inscribían como trabajadores manuales de la industria). Por otro lado, eran los titulados en estudios primarios, BUP y COU los que en mayor proporción se inscribían sin especificar demanda concreta, probablemente por no sentirse preparados para ningún trabajo en especial.

De todo el conjunto, casi el $58 \%$ tuvo experiencia laboral previa a la realización de los cursos. Trabajaron en diversas ocupaciones, pero mayoritariamente como trabajadores manuales de industria y servicios, así como en el comercio, casi siempre con baja categoría profesional (peones, etc.). Además, manifestando una elevada rotación laboral, realizaron trabajos sucesivos, hasta un máximo de siete, en general por periodos de tiempo breves. En el trabajo considerado como principal, tan sólo el $38.3 \%$ se ocupó por espacio de más de un año.

Se da una cierta correspondencia entre el nivel de formación y la categoría de los trabajos desempeñados antes de los cursos, en los niveles bajos. También se aprecia un porcentaje de infrautilización, sobre todo en el caso de los de mayor titulación, que ocupan puestos de trabajadores manuales.

Del colectivo que trabajó antes de los cursos, un alto porcentaje $(29,3 \%)$ lo hizo sin contrato laboral y de los que lo tuvieron, el $52 \%$ lo tenía temporal. Destaca, por tanto, el escaso número de trabajadores con contrato fijo o indefinido, así como la gran variedad de modalidades de contratación.

El trabajo anterior, que los alumnos destacaban como más importante, se realizó en empresas privadas, mayoritariamente, y de pequeño número de empleados, pertenecientes a los sectores de industria y servicios, sobre todo al comercio.

La jornada laboral realizada era predominantemente de 40 horas semanales, aunque destaca el significativo porcentaje que superaba ampliamente este horario, lo que indica las duras condiciones de los trabajos desempeñados, puestas aún más de manifiesto en los altos porcentajes de sueldos bajos que percibian.

Existe mayor correlación entre el nivel de formación y el sueldo recibido en el caso de las mujeres que en el de los varones, en los que destaca que los sueldos más elevados corresponden a los de menor titulación.

El trabajo lo obtuvieron, principalmente, a través de amigos y familiares $(65,3 \%)$ y es de destacar el alto porcentaje de satisfacción mostrado respecto a su trabajo anterior, basado fundamentalmente en el hecho de 
tener trabajo y en el tipo de trabajo y su ambiente; frente a la insatisfacción (sólo el $21 \%$ de los que trabajaron) que lo era por resultar peligroso, por no tener perspectivas de futuro, o por el bajo salario.

Para la mayoría de los que declaraban la causa, el trabajo concluyó por finalización del contrato.

La experiencia laboral posterior a los cursos de FPO afectaba a un porcentaje muy elevado $(83,2 \%$ encontraron trabajo). Se aprecia un claro incremento respecto a los que tuvieron trabajo anteriormente $(59,3 \%)$. En este cambio, puede haber influido, en alguna medida, el hecho de haber realizado cursos de formación ocupacional, sin olvidar, que hay otras razones que subyacen a este hecho; muy importante es el tiempo transcurrido (mayor edad, cambios en la legislación y mercado laboral), pero también se pueden establecer otras relaciones sociales, demográficas y económicas. No parece influir el género, ya que las mujeres encontraron trabajo en casi igual proporción que los varones. Sí influye la edad, pues eran los más jóvenes los que manifestaban mayor dificultad de inserción laboral; el estado civil, la situación de convivencia y dependencia parecen tener poca relación, aunque se puede afirmar que son precisamente los casados, o con personas a su cargo, los que mayores dificultades presentan para encontrar trabajo tras realizar los cursos, probablemente, por la actual precariedad del mercado laboral, que no ofrece soluciones definitivas a personas con cargas familiares, que no se sienten satisfechas con trabajos inestables o mal remunerados.

Los jóvenes que tras los cursos no encontraron empleo dedicaron su tiempo, no tanto a buscarlo como a seguir estudiando, lo que indica la necesidad que sentían de obtener mayor formación para competir en un complejo mundo laboral.

Las principales características de la experiencia laboral posterior a la realización de los cursos son las siguientes:

- Predominio de trabajadores manuales de los servicios, seguidos de los de comercio (en buena parte también manuales de los servicios) y de los de la construcción e industria (igualmente, manuales).

- La importancia del proceso de terciarización del mercado laboral, puesto de relieve por los cambios en las ocupaciones dominantes en el mercado.

- La precariedad laboral se revela en el alto grado de rotación en diversos puestos de trabajo. Son muy numerosos los jóvenes cuya experiencia laboral tras la formación recibida no se limitaba a un sólo trabajo, sino que más de la mitad había desempeñado al menos dos, y cerca de un tercio, tres, llegando algunos hasta a un total de seis empleos sucesivos. 
Aunque casi la mitad encontró trabajo antes de los seis meses desde la realización de los cursos, eran numerosos (más del 20\%) los que tardaron más de un año en hacerlo. No obstante, no mantuvieron el empleo conseguido más que en la mitad de los casos, dado que se trataba, en su mayoria, de contratos temporales y de corta duración. Al igual que en los trabajos previos a los cursos, la mayoría se empleó en empresas privadas, de pocos trabajadores, del sector de servicios, aunque existía un notable aumento de los que lo hacían en empresas de gran número de trabajadores. Los sueldos percibidos, en esta segunda etapa laboral, eran claramente más elevados que en la previa, en parte, claro está, también por el tiempo transcurrido.

La manera de obtener trabajo fue, predominantemente, a través de amigos y familiares, de forma similar a lo que ocurría en la fase previa a los cursos. También se mantenían los motivos de la satisfacción por el trabajo que, en la nueva situación, eran más por el tipo de trabajo, que por el hecho de tenerlo, lo que supone un notable cambio cualitativo.

Un pequeño grupo (el $3.3 \%$ ) del total trabajó por cuenta propia, un tercio de ellos como profesionales y el resto en trabajos de baja cualificación. Al trabajo por cuenta propia dedicaron, por término medio, más de cuarenta horas a la semana. El motivo de trabajar por cuenta propia no se derivaba tanto del espíritu de empresa, o deseo de independencia, como el no haber encontrado nada mejor. Ninguno recibió ayuda externa para su proyecto profesional particular.

En el momento de realizar la encuesta, había una parte de los jóvenes en situación de paro, motivada, en más del $40 \%$ de los casos, por la finalización del contrato (indicador de nuevo de la precariedad laboral) y un significativo $6 \%$ por quiebra de la empresa, lo que también puede ser un indicador de dificultades en algún tipo de empresas.

Al analizar la relación entre la formación lograda en los cursos y la experiencia laboral obtenemos como conclusiones más destacables:

- Pese al elevado porcentaje de jóvenes que consiguieron un trabajo posterior, éste se adecuaba poco a la formación recibida en los cursos. No obstante, tal adecuación es mayor para los más jóvenes (con menor formación), para los varones $y$, según los estudios cursados, para aquellos con un bajo nivel de formación.

- Tras la realización de los cursos continuó la precariedad en el empleo, manifestada en la excesiva rotación laboral en trabajos de escasa cualificación, circunstancia que afectaba más a los hombres que a las mujeres. 
- Se produjo una terciarización progresiva del empleo, que afectaba sobre todo a la categoria de trabajadores manuales. También aumentaron los jóvenes que alternaban trabajos en sectores productivos diferentes, lo que indica más versatilidad tras los cursos, sea por su mayor formación relativa, o por la necesidad de obtener cualquier empleo.

- Al comparar las ocupaciones que tenían antes y después de los cursos de formación recibidos, también se aprecia el aumento de la terciarización. No obstante, son mayoría los que continuaron en la misma profesión que antes de la realización de los cursos, posiblemente, por la valoración positiva de la experiencia por parte de los empresarios. Entre hombres y mujeres, existían algunas diferencias, que muestran una incidencia positiva de los cursos realizados. Así, entre los varones había un aumento considerable de los que se ocupaban en trabajos manuales de la construcción y los servicios, en clara sintonía con el elevado número de cursos de este carácter que realizaron. En el caso de las mujeres, también aumentaron las trabajadoras manuales de los servicios, comercio y técnicas, como corresponde a los cursos específicos para niveles superiores.

- Se produjo, tras los cursos, una notable mejora del tipo de contrato y de los sueldos percibidos, en los que se aprecia, además, mayor correlación con la formación.

Respecto a la valoración de los cursos de formación ocupacional realizados para integrarse en el mercado de trabajo, los propios afectados opinaban, mayoritariamente, que no les habían servido en demasía. No obstante, sí que los consideraban de utilidad, aunque ésta fuera, sobre todo, para mejorar su bagaje formativo. Resulta muy expresivo comparar las respuestas dadas a la pregunta sobre la valoración de los cursos, en dos fases distintas de la encuesta. Su opinión sobre los mismos variaba según que los consideraran por sí mismos o con relación al trabajo, dado que siendo las mismas personas y los mismos cursos se obtuvieron porcentajes diferentes de cada respuesta. Mientras que en la primera vez que se les preguntaba, el $34 \%$ decía que les fue útil para su formación, aunque no encontraran empleo, en la segunda sólo el $15,2 \%$ respondía lo mismo. En cambio, dos tercios del total manifestaban, en una segunda pregunta, que el curso les dio suficiente formación para el trabajo. Todo ello indica que existe una disociación de ideas en los jóvenes, respecto a la formación recibida en los cursos, su puesta en práctica en el trabajo y el propio desarrollo de los mismos. La crítica más importante que hacían a los cursos era su deficiente organización y la escasez de demanda, en el mercado, con la titulación que otorgan.

Como propuestas para organizar cursos de formación ocupacional, además de los que ya existían, con miras a facilitar la inserción en el mer- 
cado de trabajo, los jóvenes expresaban el deseo de que aumentasen los de carácter práctico, en colaboración con empresas, y los de materias como idiomas o informática, que coincidian, precisamente, con los que ellos mismos estudiaron como complemento formativo, de forma privada. Aún existía un porcentaje representativo que se mostraba escéptico respecto a la necesidad y utilidad de los cursos.

Las dificultades para encontrar empleo se resumen para los jóvenes en falta de formación o experiencia, discriminación por diversas causas y postura de fuerza por parte de los empleadores, ante la abundante oferta existente. Para mejorar la situación del empleo, proponían diversas medidas, que se resumen en: mejora de la legislación laboral tendente a un reparto del trabajo existente; aumento del empleo fijo y de inversiones para promover la oferta de empleo; control a los empresarios en su política de contratación laboral y eliminación de las ETT, que se consideran muy perniciosas, al contribuir a aumentar la precariedad en el empleo.

Para conseguir un empleo manifestaban una gran disponibilidad. La gran mayoría estaba dispuesta, sobre todo, a desplazarse de su lugar de origen, a aceptar cualquier trabajo y a tener un sueldo bajo, por este orden. En cambio, lo que más les inquietaba era el tipo de contrato con el que aceptar dicho empleo. Como condiciones positivas que querrían tener para trabajar la más importante era conseguir estabilidad y, en segundo lugar, que la ocupación se relacionara con la formación recibida.

\section{A MODO DE CONCLUSIÓN FINAL}

La crisis industrial de los años setenta puso el punto final a la etapa desarrolista del capitalismo, caracterizada por el uso intensivo del factor trabajo y las políticas del pleno empleo. La reorganización productiva posterior ha estado definida por la aplicación progresiva de las nuevas tecnologías de la información, que han sustituido, cada vez en mayor proporción, mano de obra por capital; la flexibilización del empleo y la puesta en práctica de nuevas modalidades contractuales (temporales, a tiempo parcial, etc.) y, finalmente, la segmentación del mercado laboral. Estas circunstancias han incidido negativamente en la disminución de la oferta de trabajo, así como en la precarización del empleo.

Esta situación de crisis del empleo estable ha afectado, de manera singular, a los jóvenes, colectivo laboral, que falto de experiencia, debe abrirse camino profesional en condiciones históricas de extrema competencia. En este marco de referencia, la formación ocupacional no reglada puede 
desempeñar un cometido de primera magnitud, como vía de capacitación de los trabajadores que han recibido una formación insuficiente. Por una parte, proveería de mano de obra con la cualificación exigida por el mercado de trabajo, mientras, por otra, incorporaría al proceso productivo a aquellos grupos que, de no ser así, quedarían marginados del mismo, por su escasa preparación y falta de conocimientos en relación con las nuevas técnicas de producción.

Sin embargo, la valoración de la política de formación ocupacional que hemos llevado a cabo en el municipio de Getafe permite matizar la eficacia que se deriva de la impartición de este tipo de enseñanza no reglada. La relación entre la formación recibida y el empleo hallado puede considerarse relativa, ya que los propios alumnos consideraban, en un elevado porcentaje, que no les sirvió para potenciar su inserción laboral. Únicamente, en el caso de los alumnos de menor nivel formativo y en los cursos de aprendizaje de oficios el éxito de la inserción laboral puede considerarse significativo.

La causa de este relativo fracaso de la formación ocupacional hay que buscarla en la grave desconexión existente entre la demanda potencial del mercado y la planificación institucional pública de la oferta formativa que intenta dar respuesta a la misma. Este tipo de formación requiere de una mayor coordinación con el mundo empresarial. No debe olvidarse, en la programación de las actividades formativas, que la demanda laboral es básica para definir los perfiles laborales que el mercado precisa. La búsqueda de un modelo mixto, con intervención directa, tanto de las instancias institucionales como de la empresa privada, se hace necesaria, si no queremos que la formación recibida se convierta en un mero entretenimiento de los parados.

\section{BIBLIOGRAFÍA}

aguilera Arilla, M.J.; Azcarate luxán, M.V.; Borderías Uribeondo, M.P.; Gonzalez Yanci, M.P.; Murillo Castillejo, J., y Santos Preciado, J.M. (1996): Desequilibrios del mercado laboral juvenil en el A.M.M. Informe del trabajo de investigación subvencionado por la Dirección General de la Juventud, C.A.M. Madrid, 527 págs.

aguilera Arilla, M.J.; Ayllón Trujillo, M.T.; Azcarate luxá, M.V.; Borderias Uaibeondo, M.P.; Gonzalez YanCi, M.P.,y Santos Preciado, J.M. (1999): La gestión de las políticas de fomento del empleo juvenil, en el escalón territorial municipal. Informe del trabajo de investigación subvencionado por la Dirección General de la Juventud, C.A.M. Madrid, 582 págs.

ARAGON y otros (1987): El mercado de trabajo en Madrid y las políticas de empleo. Consejería de Trabajo de la Comunidad de Madrid. Madrid.

Arasa Medina, C. (1988): Política de empleo en España: una propuesta de programa a medio plazo. Cuadernos de la UNED. UNED. Madrid, 123 págs.

CACHÓN, L. (1989): “Políticas de inserción laboral de los jóvenes en el mercado de trabajo de la C.E.", Revista de Estudios de la Juventud. Instituto de la Juventud de Madrid. 
CASAL, J.; MASJUAN, J.M., y PLANAS, J. (1991): La inserción social y profesional de los jóvenes. CIDE. MEC. Madrid, 337 págs.

CASAL BATLLER, J. y otros (1991): La inserción social y profesional de los jóvenes. Ministerio de Educación y Ciencia. Madrid.

Castaño Collado, C. (1994): Tecnología, empleo y trabajo en España. Alianza Editorial. Madrid, 244 págs.

EQUIPO EUSYA (1988): Paro y empleo juvenil en la periferia urbana madrileña. Consorcio Rector del Plan de Prevención de la Delincuencia y la Marginación Social en San Blas, Orcasitas y Pan Bendito. Madrid.

FIguera Gazo, P. (1996): La inserción del universitario en el mercado de trabajo. EUB, SL. Barcelona, 332 págs.

FREEMAN, C. y SOETE, L. (1996): Cambio tecnológico y empleo. Una estrategia de empleo para el siglo xxi. Fundación Universidad Empresa. Madrid, 210 págs.

Garrido, J. y Toharia, L. (1991): Prospectiva de las ocupaciones y la formación en la España de los noventa. Instituto de Estudios y Análisis Económicos. Ministerio de Economía y Hacienda. Madrid, 233 págs.

GonzÁlez AZnAR, J. (1988): "Las políticas municipales de empleo juvenil» en Revista de Estudios de la Juventud. Instituto de la Juventud. Madrid.

Mayoral Lobato, J. (1992): Mercado de trabajo, políticas de empleo y desarrollo local. Fundación Universidad Empresa. IRMASA. Madrid, 375 págs.

MONTORO, R. (1985): La inserción en la actividad económica: empleo y paro juvenil. Publicaciones de Juventud y Sociedad, S. A., Instituto de la Juventud, Ministerio de Cultura. Madrid, 191 págs.

OCDE (1987): La naturaleza del desempleo de los jóvenes. Ministerio de Trabajo y Seguridad Social. Madrid, 320 págs.

OCDE/CERI (1989): Inserción de los jóvenes en una sociedad en cambio. Editorial Narcea. Madrid, 167 págs.

Peinado López, A. (1988): La discriminación de la mujer en el mercado de trabajo español. Ministerio de Trabajo y Seguridad Social. Madrid, 198 págs.

PEIRO, J y MOTER, D. (1987): Socialización laboral y desempleo juvenil: la transición de la escuela al trabajo. Nau. Valencia.

Pérez Borrego, G. (1991): "Condiciones de trabajo de los jóvenes: de la flexibilidad laboral a la economía sumergida" en Rodriguez Ramos, M.J. (eds.), Formación profesional, contratación y mercado de trabajo. Universidad de Sevilla. Sevilla, págs. 135-146.

Pérez SÁnChez, M. (1997): Formación y empleo en España. Análisis y evaluación de la política de Formación Ocupacional. Universidad de Granada. Granada, 234 págs.

RODRIGUEZ Bolaños, T. (1988): «El papel de los ayuntamientos en la política del empleo juvenil», Revista de Economía y Sociología del Trabajo. Madrid.

Rodriguez Ramos, M. J. (eds.) (1991): Formación profesional, contratación y mercado de trabajo. Universidad de Sevilla. Sevilla, 161 págs.

ROSE, J.(1987): En busca de empleo: formación, paro y empleo. Ministerio de Trabajo. Madrid.

Santos Preciado, J. M. y otros (1987): "Mercados locales de empleo", Alfoz. Madrid, pp 30-49.

ToRregrosa, J. R. y otros (1989): Juventud, trabajo y desempleo: un análisis psicosociológico. Ministerio de Trabajo y Seguridad Social. Madrid.

VALLÉS MARTinez, M. S.; MOnCADA, A., y CALLEJO, M. (1987): La juventud ante el trabajo: nuevas actitudes en los ochenta. Tres ensayos. Popular. Madrid, 111 págs. 